\section{THU0355 PARAMETRIC CARDIAC MAGNETIC RESONANCE IMAGING IDENTIFIES ARRHYTHMOGENIC SUBSTRATES IN SYSTEMIC SCLEROSIS PATIENTS}

S. I. Mavrogeni ${ }^{1}$, L. Gargani ${ }^{2}$, A. Pepe ${ }^{3}$, L. Monti ${ }^{4}$, G. Markousis-Mavrogenis ${ }^{1}$, M. De Santis ${ }^{4}$, A. Meloni ${ }^{3}$, L. Koutsogeorgopoulou ${ }^{5}$, G. Karabela ${ }^{6}$,

E. Stavropoulos ${ }^{6}$, G. Katsifis ${ }^{6}$, K. Bratis $^{1}$, S. Bellando Randone ${ }^{7}$, S. Guiducci ${ }^{7}$, C. Bruni ${ }^{7}$, A. Moggi-Pignone ${ }^{7}$, T. Dimitroulas ${ }^{8}$, P. Voulgari ${ }^{9}$, G. Kolovou' ${ }^{1}$, V. K. Bournia ${ }^{10}$, M. Mukherjee ${ }^{11}$, J. Lima ${ }^{11}$, G. D. Kitas ${ }^{12}$, P. Sfikakis ${ }^{10}$, M. Matucci-Cerinic ${ }^{7}{ }^{1}$ Onassis Cardiac Surgery Center, Kallithea, Greece; ${ }^{2}$ Institute of Clinical Physiology - National Research Council, Pisa, Italy; ${ }^{3}$ Magnetic Resonance Imaging Unit, Fondazione G. Monasterio C.N.R, Pisa, Italy; ${ }^{4}$ Humanitas Clinical and Research Center, Rozanno (MI), Italy; ${ }^{5}$ Pathophysiology Dpt, Laikon Hospital, Athens, Greece; ${ }^{6}$ Navy Hospital, Athens, Greece; ${ }^{7}$ Department of Experimental and Clinical Medicine, Divisions of Internal Medicine and Rheumatology AOUC, University of Florence, Florence, Italy; ${ }^{8}$ Aristotle University of Thessaloniki, Thessaloniki, Greece; ${ }^{9}$ Faculty of Medicine, School of Health Sciences, University of Ioannina, 45110, Ioannina, Greece; ${ }^{10}$ First Department of Propaedeutic and Internal Medicine, Laikon Hospital, Athens University Medical School, Athens, Greece; ${ }^{11}$ Johns Hopkins University School of Medicine, Baltimore, United States of America; ${ }^{12}$ Arthritis Research UK Centre for Epidemiology, Manchester University, Manchester, United Kingdom

Background: Cardiac involvement in systemic sclerosis (SSc) accounts for $26-36 \%$ of deaths. This most frequently manifests as ventricular rhythm disturbances (VRDs), eventually culminating in sudden cardiac death. However, no specific guidelines exist for implantation of cardioverter defibrillators (ICD) in SSc patients. Parametric cardiovascular magnetic resonance (CMR) indices of myocardial oedema and fibrosis like native T1/T2 mapping have been shown to be associated with prognosis in SSc patients with acute cardiac events and normal echocardiograms. However, their relationship with arrhythmogenicity per se has not been previously investigated in SSc.

Objectives: To investigate the relationship between parametric CMR indices and arrhythmogenicity in SSc patients.

Methods: 84 consecutive SSc patients ( $80 \%$ diffuse-cutaneous SSc) from eight European centers presenting with cardiac symptoms were examined using a 1.5 T CMR system. 24h Holter recordings were obtained within a month of the CMR scan. The presence of VRDs was defined as any type of premature ventricular contraction (PVC) in couples, triplets, bigeminism, trigeminism, quadrigeminism and non-sustained ventricular tachycardia, as well as having $>30$ PVCs per hour. Logistic regression analysis was used to evaluate the relationship between VRD occurrence and native T1/T2 mapping as well as myocardial extracellular volume fraction (ECV).

Results: Mean age in the cohort was 55 (13) years and 78 (93\%) patients were female. Of these, $67(80 \%)$ experienced at least one type of VRDs. Each $10 \mathrm{~ms}$ increase of native T1-mapping was associated with a higher occurrence of VRDs [odds ratio (95\% confidence interval): 1.21 (1.08-1.36), $p=0.001$ ]. Similarly, a $1 \%$ increase in ECV conferred an increased probability of experiencing VRDs [1.25 (1.01-1.53), $p=0.037]$. Lastly, a $1 \mathrm{~ms}$ unit increase in T2-mapping also led to increased probability of having experienced VRDs [1.09 (1.01-1.19), $\mathrm{p}=0.035$ ].

Conclusion: Parametric CMR indices are associated with arrhythmogenicity in SSc patients with cardiac symptoms and should be investigated further in larger studies for their clinical utility in selecting high-risk SSc patients for ICD implantation.

Disclosure of Interests: Sophie I. Mavrogeni: None declared, Luna Gargani: None declared, Alessia Pepe: None declared, Lorenzo Monti: None declared, George Markousis-Mavrogenis: None declared, Maria De Santis: None declared, Antonella Meloni: None declared, Loukia Koutsogeorgopoulou: None declared, Georgia Karabela: None declared, Efthymios Stavropoulos: None declared, Gkikas Katsifis Grant/research support from: UCB Pharma, Janssen, Abbvie, Novartis, MSD, Aenorasis, Genesis Pharma, Pfizer, Roche, Consultant of: UCB Pharma, Janssen, Abbvie, Novartis, MSD, Aenorasis, Genesis Pharma, Pfizer, Roche, Speakers bureau: UCB Pharma, Janssen, Abbvie, Novartis, MSD, Aenorasis, Genesis Pharma, Pfizer, Roche, Konstantinos Bratis: None declared, Silvia Bellando Randone: None declared, Serena Guiducci: None declared, Cosimo Bruni: None declared, Alberto Moggi-Pignone: None declared, Theodoros Dimitroulas: None declared, Paraskevi Voulgari: None declared, Genovefa Kolovou: None declared, Vasiliki-Kalliopi Bournia Grant/research support from: Travel Grant from Boehringer Ingelheim, Monica Mukherjee: None declared, Joao Lima: None declared, George D. Kitas: None declared, Petros Sfikakis Grant/research support from: Grant/research support from Abvie, Novartis, MSD, Actelion, Amgen, Pfizer, Janssen Pharmaceutical, UCB, Marco Matucci-Cerinic Grant/ research support from: Actelion, MSD, Bristol-Myers Squibb, Speakers bureau: Acetelion, Lilly, Boehringer Ingelheim

DOI: 10.1136/annrheumdis-2020-eular.6575

\section{THU0356 \\ DISEASE ACTIVITY ASSESSMENT IN SYSTEMIC SCLEROSIS PATIENTS BASED ON THE CURRENTLY AVAILABLE ACTIVITY INDICES ANDTHE PHYSICIAN GLOBAL ASSESSMENT}

T. Minier ${ }^{1}$, V. Lóránd ${ }^{2}$, Z. Bálint ${ }^{1}$, D. Komjati ${ }^{1}$, G. Nagy ${ }^{1}$, A. Kovács ${ }^{1}$, O. Koncz ${ }^{3}$ C. Varjú ${ }^{1}$, L. Czirják' ${ }^{1}$ G. Kumanovics ${ }^{1}$, B. Nemeth. ${ }^{1}$ University of Pecs, Rheumatology and Immunology, Pecs, Hungary; ${ }^{2}$ Polyclinic of the Hospitaller Brothers of St. John of God, Budapest, Hungary; ${ }^{3}$ University of Pecs, Medical School, Pecs, Hungary

Background: Disease activity assessment is crucial in defining the appropriate therapy and to monitor the efficacy of treatment in systemic sclerosis.

Objectives: We aimed to test the performance of the 'old' European Scleroderma Trials and Research Group (EUSTAR) Activity Index (old-AI) (1), the 'new' EUSTAR activity index (new-Al) (2), and the scleroderma activity index derived from the old-Al (Pecs-Al) (3). We compared the three indices to the disease activity based on the physician's global assessment (PGA). We also assessed the correlations with the change in modified Rodnan Skin Score (MRSS), FVC and arthritis after one year follow-up.

Methods: We evaluated 77 patients (50 diffuse /dcSSc/ and 27 limited cutaneous SSc /lcSSc/ patients) from a single tertiary clinical center. Cohort enrichment was performed to increase the number of patients with early disease and dcSSc. Seventy-two patients were re-evaluated after one year. Nine patients had overlap syndromes: rheumatoid arthritis $(n=3)$, Sjögren syndrome $(n=2)$, polymyositis $(n=2)$, and mixed connective tissue disease $(n=2)$. The overall disease activity was evaluated using both composite indices (old-Al, Pecs-Al, new-Al) and the PGA of disease activity, based on the blinded evaluation of a single physician (LV). In addition to the minimal essential data from the EUSTAR database we also performed detailed assessment of the musculoskeletal involvement evaluating measures of hand function, DAS28 scores, and the Clinical Disease Activity Index (CDAI) (4).

Results: Three times more patients with active disease were identified by the new-Al compared to the old-Al at baseline investigation ( $n=37,48.7 \%$, vs $n=11,14.3 \%)$. Two patients (18\%) with active disease based on the old-Al were missed by the new-Al. Pecs-Al index identified 15 patients (19.5\%) with active disease (cut-off $>2.75$ points). Active disease was equally frequent in dcSSc and IcSSc patients based on old-Al, but was more frequent in dcSSc patients based on the new-Al in the whole cohort, and also after excluding overlap cases.

Patients with active disease based on the old-Al had more frequently rheumatoid factor (6/9, vs. $12 / 45, p=0.047)$, and $D L C O<70 \%(11 / 11$, vs. $36 / 65, p<0.01)$. Active disease based on the new-Al was associated with current cyclophosphamide treatment $(9 / 37, v s .2 / 39, p=0.023)$, and diabetes mellitus $(7 / 30$, vs. $0 / 39$, $\mathrm{p}<0.01)$. The PGA correlated moderately at both baseline and one year follow-up examination with the old-Al (rho: 0.519, and rho: 0.692, respectively, $p<0.001$ ), the new-Al (rho: 0.401, and rho: 0.429, respectively, $\mathrm{p}<0.001$ ), and the Pecs-Al (rho: 0.425 , and rho: 0.593 , respectively, $p<0.001$ ).

CDAl correlated significantly with the old-Al (rho: 0.345 , and rho: 0.283 , respectively, $\mathrm{p}<0.05$ ) and the Pecs-Al (rho: 0.363 , and rho: 0.324 , respectively, $\mathrm{p}<0.05$ ) at both the baseline and one-year follow-up investigations, but showed no consistent correlation to the new-Al or PGA.

Conclusion: The two validated disease activity indices indentify different patient groups. Joint involvement is potentially underrepresented in the new EUSTAR activity index. Active disease is also present in IcSSc and should be assessed regularly in these patients.

\section{References:}

[1] Valentini G, et al. Ann Rheum Dis 2003; 62: 901-3.

[2] Valentini G, et al. Ann Rheum Dis 2017;76:270-276.

[3] Minier T, et al. Rheumatology (Oxford) 2010;49(6):1133-45.

[4] Lorand V, et al. Rheumatology (Oxford). 2016;55(10):1849-58

Acknowledgments: This work was supported by the EU Seventh Framework Program [FP7/2007-2013] under Grant Agreement $n^{\circ} 305495$ (DeSScipher), by the Hungarian Scientific Research Fund (contract $n^{\circ}: 112939$ ), and the EU under the Grant Agreement $n^{\circ}$ PEPSYS GINOP-232-15-2016-00050.

Disclosure of Interests: Tünde Minier Speakers bureau: Actelion, Abbvie, MSD, Pfizer, Lilly, Roche, Veronika Lóránd: None declared, Zsófia Bálint: None declared, Dalma Komjati: None declared, Gabriella Nagy Speakers bureau: MSD, Antonietta Kovács: None declared, Orsolya Koncz: None declared, Cecília Varjú Consultant of: Boehringer Ingelheim RCV GmbH \& Co KG, Speakers bureau: Lilly, László Czirják Consultant of: Actelion, BI, Roche-Genentech, Lilly, Medac, Novartis, Pfizer, Bayer AG, Gabor Kumanovics Consultant of: Boehringer, Teva, Speakers bureau: Roche, Lilly, Novartis, Balazs Nemeth: None declared

DOI: 10.1136/annrheumdis-2020-eular.2976 